\title{
ON AN ASYMPTOTIC EXPANSION OF THE KONTOROVICH-LEBEDEV TRANSFORM
}

\section{Naylor}

ABSTRACT. An asymptotic expansion valid for large positive values of $s$ is constructed for the integral transform

$$
F(s)=\int_{0}^{\infty} K_{i s}(x) f(x) \frac{d x}{x}
$$

where $K_{i s}(x)$ denotes the modified Bessel function of the third kind of purely imaginary order.

\section{Introduction}

In an earlier paper [2], a formula was developed that gave the form as $s \rightarrow \infty$ of the Kontorovich-Lebedev transform

$$
F(s)=\int_{0}^{\infty} K_{i s}(x) f(x) \frac{d x}{x}
$$

where $K_{i s}(x)$ denotes the Macdonald-type Bessel function defined by the equation [1, p. 85]

$$
K_{i s}(x)=\int_{0}^{\infty} e^{-x \cosh t} \cos (s t) d t .
$$

As will be explained shortly, the results derived in [2] were confined to classes of functions $f(x)$ that exhibited exponential growth at infinity. The present investigation is devoted to the determination of the asymptotic form of the transform $F(s)$ of functions $f(x)$ that are exponentially damped at infinity.

The only other investigation of the asymptotic form of the Kontorovich-Lebedev transform appears to be that of Wong [6] who considered the closely related, but reciprocal, problem of determining the form of $f(x)$ as $x \rightarrow \infty$ from that of $F(s)$ in the cases where, in the present notation, the asymptotic form of the product $e^{s \pi} F(s)$ was exponentially damped, oscillatory, or purely algebraic at infinity. Wong [6] also considered the relation connecting the forms of $F(s)$ and $f(x)$ as $s \rightarrow 0$ and $x \rightarrow 0$, respectively.

The formula obtained in [2] applies to functions $f(x)$ that tend to zero as $x \rightarrow 0$ and are such that

$$
f(x) \sim e^{x \cos \gamma} \sum_{n=0}^{\infty} c_{n} x^{1-n-c}, \quad x \rightarrow \infty,
$$

where $\gamma$ is real or complex, $0 \leq R e(\cos \gamma) \leq 1$, and $0<c \leq 1$ (or $\frac{1}{2}<c \leq 1$ if $\gamma=0)$. The restriction $\operatorname{Re}(\cos \gamma) \leq 1$ is necessary to ensure that the integral (1.1) is convergent at infinity, but the condition $\operatorname{Re}(\cos \gamma) \geq 0$ was required to justify the

Received April 19, 1995, revised November 6, 1995.

1991 Mathematics Subject Classification. 41A60, 44A15, 33C10.

Key words and phrases: asymptotic expansion, Kontorovich-Lebedev transform. 
method adopted in [2]. The latter condition means that the formulas obtained in [2] apply only to functions possessing exponential growth at infinity, possibly incorporating an oscillatory factor, or to functions that are purely oscillatory at infinity, but not to functions that are exponentially damped there. It is the purpose of the present paper to consider the latter case, i.e., to determine the form of $F(s)$ as $s \rightarrow+\infty$ when $\cos \gamma$ in $(1.3)$ is such that $-1<\operatorname{Re}(\cos \gamma)<0$. The method followed in [2] depended on regarding the integral (1.2) as one along the real axis of the complex $t$-plane and deforming the path of integration in a suitable manner. It has not been possible to adapt this method to deal with functions exponentially damped at infinity and a different process had to be sought. This process involves the introduction of an auxiliary function $g(x)$ related to $f(x)$ by means of the equation

$$
g(x)=x \int_{0}^{\infty} f(t)\left(x^{2}+t^{2}\right)^{-\frac{1}{2}} K_{1}\left[\left(x^{2}+t^{2}\right)^{\frac{1}{2}}\right] d t
$$

where $K_{1}$ denotes the Macdonald-type Bessel function of order unity. The utility of this formula, which is discussed in full in the next section of this paper, is that if the behavior of $f(x)$ at infinity is as described by equation (1.3), then the behavior of $g(x)$ is given by an equation of the form

$$
g(x) \sim e^{-x \sin \gamma} \sum_{n=0}^{\infty} a_{n} x^{1-n-c}
$$

as $x \rightarrow \infty$ where the coefficients $a_{n}$ can be determined in terms of the coefficients $c_{n}$ appearing in (1.3). Suppose for the moment that $\gamma$ is real and $0<\gamma<\frac{\pi}{2}$, then (1.5) shows that the function $g(x)$ will possess precisely the exponentially damped behavior at infinity that was ruled out in the earlier investigation. Furthermore, it will be shown that the Kontorovich-Lebedev transform $G(s)$ of $g(x)$ is related to that of $f(x)$ by means of the simple relation

$$
G(s)=\frac{\pi}{2} F(s) \operatorname{sech}\left(\frac{s \pi}{2}\right) .
$$

By inserting the known asymptotic formula for $F(s)$, as derived in [2], into this equation, the behavior of the transform of the exponentially damped function $g(x)$ can be found.

\section{The auxiliary function}

It will be assumed that $f(x)$ is continuous for $x>0$, satisfies (1.3), and is $O\left(x^{b}\right)$ as $x \rightarrow 0$ where $b>0$. First, the formula (1.6) is obtained. The origin of this formula lies in the equation

$$
t \int_{0}^{\infty}\left(x^{2}+t^{2}\right)^{-\frac{1}{2}} K_{i s}(x) K_{1}\left[\left(x^{2}+t^{2}\right)^{\frac{1}{2}}\right] d x=\frac{\pi}{2} K_{i s}(t) \operatorname{sech}\left(\frac{s \pi}{2}\right) .
$$

This equation is established in the Appendix. Upon multiplying both sides of (2.1) by $t^{-1} f(t)$ and integrating for $0<t<\infty$, it is found after a change in the order of integration that

$$
\int_{0}^{\infty} K_{i s}(x) \int_{0}^{\infty} f(t)\left(x^{2}+t^{2}\right)^{-\frac{1}{2}} K_{1}\left[\left(x^{2}+t^{2}\right)^{\frac{1}{2}}\right] d t d x=\frac{\pi}{2} F(s) \operatorname{sech}\left(\frac{s \pi}{2}\right) .
$$

To justify the change in the order of integration, it will be verified that the repeated integral on the left-hand side of (2.2) is absolutely convergent. With this aim in view, 
we note first that the function $K_{1}(x)$ is $O\left(x^{-1}\right)$ near $x=0$ and $O\left(x^{-\frac{1}{2}} e^{-x}\right)$ at infinity, so that the inner integral on the left-hand side of (2.2) does not exceed the quantity

$$
C_{1} \int_{0}^{1}\left(x^{2}+t^{2}\right)^{-1}|f(t)| d t+C_{2} \int_{1}^{\infty}\left(x^{2}+t^{2}\right)^{-\frac{3}{4}} e^{-t}|f(t)| d t
$$

in absolute magnitude where $C_{1}, C_{2}$ are constants. Since $f(t)$ is $O\left(t^{b}\right)$ at the origin and $\left(t^{1-c} e^{t \cos \gamma}\right)$ at infinity, the expression (2.3) does not exceed

$$
C_{1}^{\prime} \int_{0}^{1} t^{b}\left(x^{2}+t^{2}\right)^{-1} d t+C_{2}^{\prime} \int_{1}^{\infty} t^{-\frac{1}{2}-c} e^{-t(1-\cos \gamma)} d t
$$

where $C_{1}^{\prime}, C_{2}^{\prime}$ are constants. It is easily shown that the first integral in (2.4) is $O\left(x^{-2}\right)$ if $x$ is large and, by subdividing the interval at the point $x, O\left(x^{b-1}\right)+O(1)$ if $b \neq 1$ and $x$ is small, or $O(\log x)$ if $b=1$ and $x$ is small. The second integral in (2.4) is independent of $x$ and convergent. It follows from these results and the fact that $\left|K_{i s}(x)\right| \leq K_{0}(x)$ that if $b \neq 1$, then the modulus of the expression on the left-hand side of (2.2) is less than the quantity

$$
A \int_{0}^{1} x^{b-1} K_{0}(x) d x+B \int_{1}^{\infty} x^{-2} K_{0}(x) d x+C \int_{0}^{\infty} K_{0}(x) d x
$$

where $A, B, C$ are constants. Since $b>0$, all integrals in (2.5) exist. If $b=1$, then the $x^{b-1}$ factor in the first integral in (2.5) is replaced by $\log x$, and the resulting integral is convergent. Hence, in either case, the integral on the left-hand side of (2.2) is absolutely convergent, so that (2.2) is established.

Upon recalling the definition (1.4) of $g(x)$, it is seen that equation (2.2) can be written as the equation

$$
\int_{0}^{\infty} K_{i s}(x) g(x) \frac{d x}{x}=\frac{\pi}{2} F(s) \operatorname{sech}\left(\frac{s \pi}{2}\right) .
$$

This equation is equivalent to (1.6) since, by definition, $G(s)$ is equal to the integral on the left-hand side of (2.6).

\section{The asymptotic expansion}

In this section, the following result is established.

Theorem. Let $g(x)$ be continuous for $0 \leq x<\infty$, be $O\left(x^{b}\right)$ as $x \rightarrow 0$ where $b>0$, and satisfy

$$
g(x) \sim e^{-x \sin \gamma} \sum_{n=0}^{\infty} a_{n} x^{1-n-c}
$$

for $x \rightarrow \infty$ where $0<\gamma<\frac{\pi}{2}$ and $0<c<1$. Let $g^{(m)}(x)$ be continuous for $0 \leq x<\infty$, be $O\left(x^{1-c} e^{-x \sin \gamma}\right)$ as $x \rightarrow \infty$, and let $x^{m+2} g^{(m)}(x) \rightarrow 0$ as $x \rightarrow 0$ for $m=1,2, \ldots$. Then for large positive values of $s$ the transform

$$
G(s)=\int_{0}^{\infty} g(x) K_{i s}(x) \frac{d x}{x}
$$

possesses the asymptotic expansion,

$$
G(s) \sim 2^{-\frac{1}{2}} \pi^{\frac{5}{2}} e^{-\frac{3}{2} s \pi}(\tan \gamma)^{1-c} \sum_{n=0}^{\infty} c_{n}(\sin \gamma)^{n+c-\frac{1}{2}} P_{i s-\frac{1}{2}}^{\frac{1}{2}-n-c}(-\cos \gamma)
$$

where the coefficients $c_{n}$ are determined in terms of the coefficients $a_{n}$ by means of the equations (3.15), (3.16) below. 
To analyse (1.4), we suppose the $\gamma$ in (1.3) is real, $0<\gamma<\frac{\pi}{2}$, and split the domain of integration into the parts $(0, T x)$ and $(T x, \infty)$ where $T$ is a positive constant. We write $\cos \gamma=\tanh \beta, T=\sinh u_{0}$, choose $T$ so that $0<u_{0}<\beta$, and set

$$
f(t)=e^{t \cos \gamma}\left[\sum_{n=0}^{N-1} c_{n} t^{1-n-c}+f_{N}(t)\right]
$$

where $f_{N}(t)=O\left(t^{1-N-c}\right)$ as $t \rightarrow \infty$, and define

$$
\begin{aligned}
& I_{1}=\int_{0}^{T x} f(t)\left(x^{2}+t^{2}\right)^{-\frac{1}{2}} K_{1}\left[\left(x^{2}+t^{2}\right)^{\frac{1}{2}}\right] d t, \\
& I_{2}=\int_{T x}^{\infty} f(t)\left(x^{2}+t^{2}\right)^{-\frac{1}{2}} K_{1}\left[\left(x^{2}+t^{2}\right)^{\frac{1}{2}}\right] d t .
\end{aligned}
$$

On substituting the expression (3.3) for $f(t)$ into (3.5) and setting $t=x \sinh u$ in the integral, it follows that

$$
I_{2}=\sum_{n=0}^{N-1} c_{n} x^{1-n-c} \int_{u_{0}}^{\infty} e^{x \cos \gamma \sinh u} K_{1}(x \cosh u)(\sinh u)^{1-n-c} d u+R_{N}(x)
$$

where

$$
R_{N}(x)=\int_{u_{0}}^{\infty} e^{x \cos \gamma \sinh u} K_{1}(x \cosh u) f_{N}(x \sinh u) d u .
$$

It is now necessary to appeal to the following formula which gives the behavior of the Bessel function when the argument is large [1, p. 135],

$$
K_{1}(x)=e^{-x}\left[\sum_{m=0}^{N-1} b_{m} x^{-m-\frac{1}{2}}+\phi_{N}(x)\right]
$$

where $\phi_{N}(x)=O\left(x^{-N-\frac{1}{2}}\right)$ as $x \rightarrow \infty$, and

$$
b_{m}=\frac{\pi^{\frac{1}{2}} 2^{-m-\frac{1}{2}} \Gamma\left(m+\frac{3}{2}\right)}{m ! \Gamma\left(\frac{3}{2}-m\right)} .
$$

Upon inserting (3.8) into (3.6), it is found that

$$
\begin{array}{r}
I_{2}=\sum c_{n} b_{m} x^{\frac{1}{2}-n-m-c} \int_{u_{0}}^{\infty}(\sinh u)^{1-n-c}(\cosh u)^{-m-\frac{1}{2}} e^{x(\cos \gamma \sinh u-\cosh u)} d u \\
+R_{N}(x)+S_{N}(x)
\end{array}
$$

where the summation in (3.9) is extended over $n=0,1, \ldots, N-1$ and $m=0,1, \ldots$, $N-1$ and

$$
S_{N}(x)=\sum_{n=0}^{N-1} c_{n} x^{1-n-c} \int_{u_{0}}^{\infty}(\sinh u)^{1-n-c} \phi_{N}(x \cosh u) e^{x(\cos \gamma \sinh u-\cosh u)} d u .
$$

To estimate the value of the integral in (3.9) when $x$ is large, we note that $\cos \gamma=$ $\tanh \beta, \sin \gamma=\operatorname{sech} \beta$, and the exponent equals $-x \operatorname{sech} \beta \cosh (u-\beta)$, so the integral in question is

$$
I=\int_{u_{0}}^{\infty} e^{-x \operatorname{sech} \beta \cosh (u-\beta)}(\sinh u)^{1-n-c}(\cosh u)^{-m-\frac{1}{2}} d u .
$$

An asymptotic expansion of this integral may be found by applying the Laplace method, the dominant contribution arising from the vicinity of the point $u=\beta$ where 
the exponential factor is greatest. Following Olver [3, p. 85], we set $v^{2}=\cosh (u-\beta)-1$ and expand the integrand in powers of $v$ to obtain

$$
I=2 e^{-x \sin \gamma} \int_{-v_{0}}^{\infty} e^{-x v^{2} \sin \gamma} h(v) v d v
$$

where

$$
\begin{aligned}
h(v) & =(\sinh u)^{1-n-c}(\cosh u)^{-m-\frac{1}{2}} \operatorname{cosech}(u-\beta) \\
& =(\sinh \beta)^{1-n-c}(\cosh \beta)^{-m-\frac{1}{2}} \sum_{k=0}^{\infty} h_{k}(m, n) v^{k-1}
\end{aligned}
$$

and $v_{0}$ is the positive root of the equation $v_{0}^{2}=\cosh \left(u_{0}-\beta\right)-1$. The coefficients $h_{k}(m, n)$ in (3.12) also depend on $\beta$, but for brevity, this dependence is not displayed explicitly. Upon estimating $I$ by the Laplace method, we find the formula

$$
I \sim 2 e^{-x \sin \gamma}(\sinh \beta)^{1-n-c}(\cosh \beta)^{-m-\frac{1}{2}} \sum_{k=0}^{\infty} h_{2 k}(m, n) \Gamma\left(k+\frac{1}{2}\right)(x \sin \gamma)^{-k-\frac{1}{2}}
$$

as $x \rightarrow \infty$. It is noted that only even-order coefficients appear in this formula; expressions for the first three such coefficients $h_{0}(m, n), h_{2}(m, n), h_{4}(m, n)$ are given in the Appendix. In view of the relation $\sin \gamma \cosh \beta=1$, the insertion of the above expression $I$ for the integral occurring in (3.9) leads to the equation

$$
\begin{aligned}
I_{2}=2 x^{-c} e^{-x \sin \gamma} \sum b_{m} c_{n} h_{2 k}(m, n) & \Gamma\left(k+\frac{1}{2}\right)(\sinh \beta)^{1-n-c}(\cosh \beta)^{k-m} x^{-m-n-k} \\
& +R_{N}(x)+S_{N}(x)+O\left(x^{-N-c} e^{-x \sin \gamma}\right)
\end{aligned}
$$

where the summation is extended over $m, n, k=0,1 \ldots, N-1$ such that $m+n+k \leq$ $N-1$. It is now necessary to obtain bounds on the quantities $R_{N}$ and $S_{N}$ appearing in (3.13); in fact, it will be shown that both of these quantities are $O\left(x^{-N-c} e^{-x \sin \gamma}\right)$ for $x \rightarrow \infty$. To estimate $R_{N}$, we recall that $f_{N}=O\left(t^{1-N-c}\right)$ and $K_{1}(t)=O\left(t^{-\frac{1}{2}} e^{-t}\right)$ for large $t$. Upon inserting these bounds into (3.7), we find that, if $x$ is large and $C$ a constant, then

$$
\left|R_{N}(x)\right| \leq C x^{\frac{1}{2}-c-N} \int_{u_{0}}^{\infty} e^{x(\cos \gamma \sinh u-\cosh u)}(\sinh u)^{1-c-N} d u .
$$

This integral is similar to that in (3.11) and is found to be $O\left(x^{-\frac{1}{2}} e^{-x \sin \gamma}\right)$, so that $R_{N}$ is $O\left(x^{-c-N} e^{-x \sin \gamma}\right)$ as $x \rightarrow \infty$. The quantity $S_{N}$ defined by (3.10) may be treated by a similar method. Upon using the bound $\phi_{N}=O\left(x^{-N-\frac{1}{2}}\right)$, it is found that $S_{N}=O\left(x^{-N-c} e^{-x \sin \gamma}\right)$.

Finally it is necessary to consider $I_{1}$ for which purpose the integral (3.4) is decomposed into the parts corresponding to the intervals $(0,1)$ and $(1, T x)$. On using the bound $f(t)=O\left(t^{1-c} e^{t \cos \gamma}\right)$ over the second interval together with the bound $K_{1}(t)=O\left(t^{-\frac{1}{2}} e^{-t}\right)$, it is found that

$$
\begin{aligned}
\left|I_{1}\right| & \leq C_{1} x^{-\frac{3}{2}} e^{-x} \int_{0}^{1}|f(t)| d t+C_{2} \int_{1}^{T x} t^{1-c}\left(x^{2}+t^{2}\right)^{-\frac{3}{4}} e^{t \cos \gamma-\left(x^{2}+t^{2}\right)^{\frac{1}{2}}} d t \\
& =0\left(x^{-\frac{3}{2}} e^{-x}\right)+x^{\frac{1}{2}-c} \int_{0}^{u_{0}}(\sinh u)^{1-c}(\cosh u)^{-\frac{1}{2}} e^{-x \sin \gamma \cosh (\beta-u)} d u
\end{aligned}
$$

after setting $t=x \sinh u, \cos \gamma=\tanh \beta, \sin \gamma=\operatorname{sech} \beta$ in the second integral. Since $0<u_{0}<\beta$, the greatest value of $-\cosh (\beta-u)$ in the interval $0<u<u_{0}$ occurs at 
$u=u_{0}$, it follows that

$$
I_{1}=O\left(x^{-\frac{3}{2}} e^{-x}\right)+O\left[x^{\frac{1}{2}-c} e^{-x \sin \gamma \cosh \left(\beta-u_{0}\right)}\right] .
$$

Since $u_{0}$ is chosen less than $\beta$, both of the exponential factors in the above bound for $I_{1}$ are less than the exponential factor in the expression (3.13) for $I_{2}$, and on setting $r=m+n+k$ in the latter, it is found that the final expression for $g(x)=x\left(I_{1}+I_{2}\right)$ takes the form

$$
g(x) \sim 2 x^{1-c} e^{-x \sin \gamma}(\sinh \beta)^{1-c} \sum_{r=0}^{\infty} a_{r} x^{-r}
$$

where

$$
a_{r}=\sum b_{m} c_{n}(\sinh \beta)^{-n}(\cosh \beta)^{r-n-2 m} \Gamma\left(r-m-n+\frac{1}{2}\right) h_{2(r-m-n)}(m, n),
$$

the summation in (3.15) extending over all non-negative integer values of $m, n$ such that $0 \leq m+n \leq r$. Since $a_{r}$ is a linear combination of $\left(c_{0}, \ldots, c_{r}\right), a_{0}$ is a multiple of $c_{0}, a_{1}$ is a linear combination of $\left(c_{0}, c_{1}\right), a_{2}$ is a linear combination of $\left(c_{0}, c_{1}, c_{2}\right)$, and so on. By solving these equations in turn, we obtain $c_{1}$ in terms of $\left(a_{0}, a_{1}\right), c_{2}$ in terms of $\left(a_{0}, a_{1}, a_{2}\right)$, etc. The first three coefficients are

$$
\begin{gathered}
c_{0}=\frac{2}{\pi} a_{0}, \quad c_{1}=\frac{2}{\pi}\left[a_{1} \sinh \beta-\frac{1}{2} c(c-1) a_{0} \operatorname{coth} \beta \cosh ^{2} \beta\right], \\
c_{2}=\frac{2}{\pi}\left[a_{2} \sinh ^{2} \beta-\frac{1}{2} c(c+1) a_{1}+\frac{1}{8} c\left(c^{2}-1\right)\left\{4+(c-2) \operatorname{coth}^{2} \beta\right\} a_{0} \cosh ^{4} \beta\right] .
\end{gathered}
$$

The result derived in [2] giving the asymptotic form of the transform $F(s)$ of the function $f(x)$ satisfying (1.3) was expressed as the asymptotic series

$$
F(s) \sim 2^{\frac{1}{2}} \pi^{\frac{3}{2}} e^{-s \pi} \sum_{n=0}^{\infty} c_{n}(\sin \gamma)^{n+c-\frac{1}{2}} P_{i s-\frac{1}{2}}^{\frac{1}{2}-n-c}(-\cos \gamma)
$$

for $s \rightarrow \infty$. The asymptotic behavior of the transform $G(s)$ of the function $g(x)$ satisfying (3.14) is obtained at once by inserting (3.17) into the relation (1.6). After writing $\cosh \left(\frac{1}{2} s \pi\right) \sim \frac{1}{2} \exp \left(\frac{1}{2} s \pi\right)$, we obtain the desired asymptotic series

$$
G(s) \sim 2^{\frac{1}{2}} \pi^{\frac{5}{2}} e^{-\frac{3}{2} s \pi} \sum_{n=0}^{\infty} c_{n}(\sin \gamma)^{n+c-\frac{1}{2}} P_{i s-\frac{1}{2}}^{\frac{1}{2}-n-c}(-\cos \gamma)
$$

for $s \rightarrow \infty$.

To obtain the equations in the form in which they appear in the Theorem, the function $g(x)$ appearing in (3.14) must be divided by the constant $2(\sinh \beta)^{1-c}$, so that (3.14) reduces to (3.1). The function $G(s)$ also must be divided by the same constant which also equals $2(\cot \gamma)^{1-c} \operatorname{since} \sinh \beta=\cot \gamma$. On dividing (3.18) by this factor, we find that the latter expansion reduces to the formula (3.2) stated in the Theorem.

The leading term of (3.2) may be expressed in a simpler form involving elementary functions only by appealing to the formula [2, p. 252]

$$
\begin{aligned}
P_{i s-\frac{1}{2}}^{\frac{1}{2}-c}(-\cos \gamma) & \sim \frac{\Gamma(1+i s-c)}{\Gamma(1+i s)}\left[\frac{2}{\pi \sin \gamma}\right]^{\frac{1}{2}} \sin \left[i s(\pi-\gamma)+\frac{1}{2}(1-c) \pi\right] \\
& \sim(2 \pi \sin \gamma)^{-\frac{1}{2}} s^{-c} e^{s(\pi-\gamma)}
\end{aligned}
$$


as $s \rightarrow \infty$, since $\Gamma(1+i s-c) / \Gamma(1+i s) \sim(i s)^{-c}$. Retention of only the dominant term in (3.2) shows that

$$
G(s) \sim \frac{1}{2} \pi^{2} c_{0} s^{-c}(\cos \gamma)^{c-1} e^{-s\left(\gamma+\frac{1}{2} \pi\right)}
$$

as $s \rightarrow \infty$ where $c_{0}=(2 / \pi) a_{0}$. More generally, if $g(x) \sim a_{N} x^{1-c-N} e^{-x \sin \gamma}$ as $x \rightarrow \infty$, it can be shown that

$$
G(s) \sim \frac{1}{2} \pi^{2} c_{N} s^{-c-N} e^{-s\left(\gamma+\frac{1}{2} \pi\right)}(\sin \gamma)^{N}(\cos \gamma)^{c-1}
$$

where $c_{N}=(2 / \pi) a_{N}(\cot \gamma)^{N}$.

\section{Oscillatory behavior at infinity}

By permitting $\sin \gamma$ in (3.1) to take complex values, the behavior of $g(x)$ at infinity can, subject to certain limitations, be made to incorporate an oscillatory factor. In this case, the integral in (3.11) is estimated by means of the saddle-point method. We proceed as before by writing $\cos \gamma=\tanh \beta$ where $\beta=\beta_{1}+i \beta_{2}$ is complex, and set $T=\sinh u_{0}$ where $u_{0}$ is chosen in accordance with the condition (4.12) derived later in this section. In addition, the quantities $\beta_{1}, \beta_{2}$ are chosen so that $\beta_{1}>0,\left|\beta_{2}\right|$ is acute, and

$$
\tanh \beta_{1} \tan ^{2} \beta_{2}<1 .
$$

This inequality follows from the equation $\cos \gamma=\tanh \left(\beta_{1}+i \beta_{2}\right)$ after separating real and imaginary parts and imposing the requirement that $0<\operatorname{Re}(\cos \gamma)<1$.

For the saddle-point method to be successful, it is necessary that the path of integration in the complex $u$-plane be deformed into a curve $C$ passing through the saddle point $u=\beta$ such that the real part of $\operatorname{sech} \beta[\cosh (u-\beta)-1]$ is strictly positive at all points of $C$, except at the saddle point. To investigate this requirement, it is convenient to set $\operatorname{sech} \beta=R e^{-i \theta}$ where $R>0$ and $|\theta|$ is acute, and examine the curves $P\left(u_{1}, u_{2}\right)=\operatorname{Re} \operatorname{sech} \beta[\cosh (u-\beta)-1]=0$ where $u=u_{1}+i u_{2}$. It is found that

$$
\begin{aligned}
P\left(u_{1}, u_{2}\right)=R \cos \theta\left[\cosh \left(u_{1}-\beta_{1}\right) \cos \left(u_{2}-\beta_{2}\right)\right. & \\
& \left.+\sinh \left(u_{1}-\beta_{1}\right) \sin \left(u_{2}-\beta_{2}\right) \tan \theta-1\right] .
\end{aligned}
$$

This equation can be written in the alternative form

$$
P\left(u_{1}, u_{2}\right)=2 R \cos \theta P_{1}\left(u_{1}, u_{2}\right) P_{2}\left(u_{1}, u_{2}\right)
$$

where

$$
\begin{aligned}
& P_{1}\left(u_{1}, u_{2}\right)=\sinh \frac{1}{2}\left(u_{1}-\beta_{1}\right) \cos \frac{1}{2}\left(u_{2}-\beta_{2}\right)-\cosh \frac{1}{2}\left(u_{1}-\beta_{1}\right) \sin \frac{1}{2}\left(u_{2}-\beta_{2}\right) \tan \frac{1}{2} \epsilon, \\
& P_{2}\left(u_{1}, u_{2}\right)=\sinh \frac{1}{2}\left(u_{1}-\beta_{1}\right) \cos \frac{1}{2}\left(u_{2}-\beta_{2}\right)+\cosh \frac{1}{2}\left(u_{1}-\beta_{1}\right) \sin \frac{1}{2}\left(u_{2}-\beta_{2}\right) \cot \frac{1}{2} \epsilon,
\end{aligned}
$$

where $\epsilon=\frac{1}{2} \pi-\theta$. It follows that $P$ vanishes on the curves $C_{1}, C_{2}$ having the equations

$$
\begin{array}{ll}
C_{1}: & \tanh \frac{1}{2}\left(u_{1}-\beta_{1}\right)=\tan \frac{1}{2} \epsilon \tan \frac{1}{2}\left(u_{2}-\beta_{2}\right), \\
C_{2}: & \tanh \frac{1}{2}\left(u_{1}-\beta_{1}\right)=-\cot \frac{1}{2} \epsilon \tan \frac{1}{2}\left(u_{2}-\beta_{2}\right) .
\end{array}
$$

It is sufficient to examine the form of these curves in the strip $-\infty<u_{1}<\infty$, $-\pi+\beta_{2}<u_{2}<\pi+\beta_{2}$. In this strip, $C_{1}$ and $C_{2}$ intersect at the saddle point $\left(\beta_{1}, \beta_{2}\right)$ and are asymptotic to the lines $u_{2}=\beta_{2} \pm \pi$. Along $C_{1}, u_{2}$ is a monotone increasing function of $u_{1}$, and along $C_{2}, u_{2}$ is a monotone decreasing function of $u_{1} \cdot C_{1}$ and $C_{2}$ divide the strip into four regions $D_{1}, D_{2}, D_{3}, D_{4}$ such that $P \geq 0$ in $D_{1}$ and $D_{3}$ and $P \leq 0$ in $D_{2}$ and $D_{4}$, as depicted in Figure 1 . 


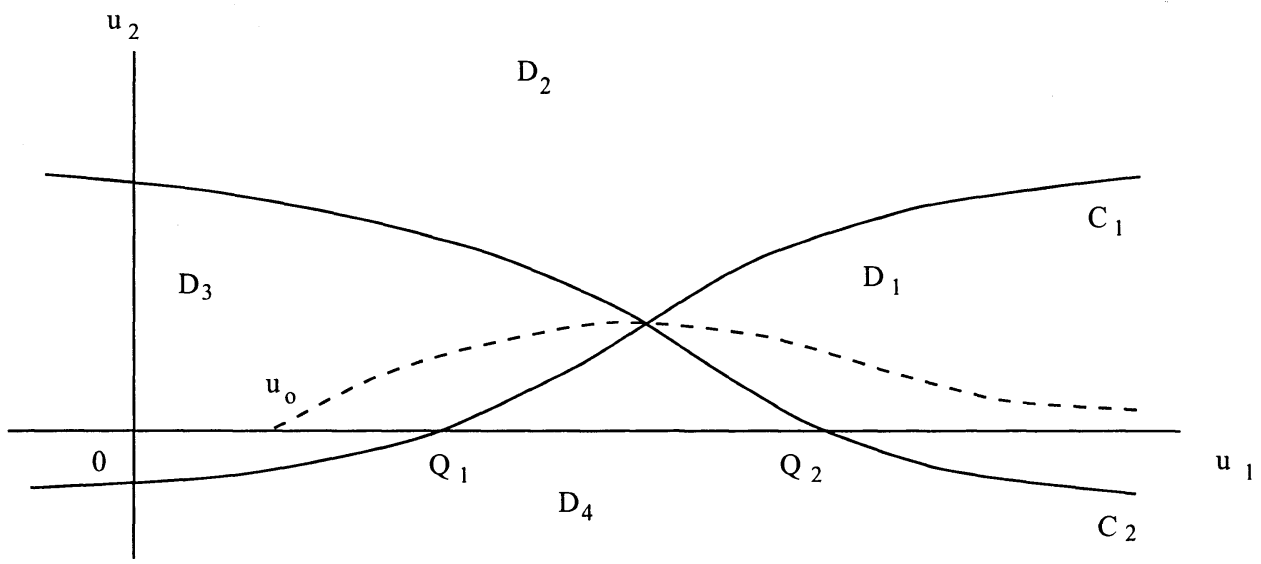

Figure 1 . Curves of $P\left(u_{1}, u_{2}\right)=0$.

To apply the saddle point method, the integral (3.10) is taken along a path coinciding with the segment $\left(u_{0}, \infty\right)$ of the real axis of the complex $u$-plane. This path must be capable of being deformed onto one that passes through the saddle point, lies wholly in the zones $D_{1}$ and $D_{3}$, and, except for the portion in the neighbourhood of the saddle point, is bounded away from the boundaries of these zones. These requirements imply that the starting point $\left(u_{0}, 0\right)$ lies in a region where $P>0$, i.e., in $D_{3}$, which, since $u_{0}>0$, implies that $C_{1}$ crosses the real axis at a point $Q_{1}\left(u_{1}^{\prime}, 0\right)$ for which $0<u_{0}<u_{1}^{\prime}$. On setting $u_{2}=0$ in (4.2), it is found that $C_{1}$ and $C_{2}$ intersect the real axis in the points $Q_{1}\left(u_{1}^{\prime}, 0\right), Q_{2}\left(u_{1}^{\prime \prime}, 0\right)$ where $u_{1}^{\prime}, u_{1}^{\prime \prime}$ satisfy the equation

$$
\cosh \left(u_{1}-\beta_{1}\right) \cos \beta_{2}-\sinh \left(u_{1}-\beta_{1}\right) \sin \beta_{2} \tan \theta=1 .
$$

To discuss the values of $u_{1}$ that satisfy this equation, we separate the real and imaginary parts of $R \cosh \left(\beta_{1}+i \beta_{2}\right)=e^{i \theta}$ giving the equations

$$
R \cosh \beta_{1} \cos \beta_{2}=\cos \theta, \quad R \sinh \beta_{1} \sin \beta_{2}=\sin \theta .
$$

It follows that

$$
\tan \theta=\tanh \beta_{1} \tan \beta_{2} .
$$

According to (4.1), we may set

$$
\tanh \delta=\tanh \beta_{1} \tan ^{2} \beta_{2}
$$

where $\delta>0$, so that by $(4.7)$,

$$
\tanh \delta=\tan \theta \tan \beta_{2} .
$$

With the aid of this relation, equation (4.5) can be written in the form

$$
\cosh \left(u_{1}-\beta_{1}-\delta\right)=\cosh \delta \sec \beta_{2} .
$$

If we now define $\sigma>0$ by the equation

$$
\cosh \sigma=\cosh \delta \sec \beta_{2},
$$

then (4.10) implies that $u_{1}=\beta_{1}+\delta-\sigma$ for $\left(Q_{1}\right)$ or $u_{1}=\beta_{1}+\delta+\sigma$ (for $Q_{2}$ ), so that the condition $0<u_{0}<u_{1}$ for $Q_{1}$ requires that $u_{0}$ be chosen so that

$$
0<u_{0}<\beta_{1}+\delta-\sigma
$$


where $\delta$ is given by (4.8) and $\sigma$ by (4.11). It follows from (4.12) that $\cosh \left(\beta_{1}+\delta\right)>$ $\cosh \sigma$, which in view of (4.11) implies that

$$
\cosh \left(\beta_{1}+\delta\right)>\cosh \delta \sec \beta_{2} .
$$

This relation may be simplified with the aid of (4.7) and (4.8) to give the inequality

$$
\cos ^{2} \theta<\cosh \beta_{1} \cos \beta_{2} \text {. }
$$

It follows from this inequality and the first equation of (4.6) that

$$
0<R \cos \theta<1 \text {. }
$$

Since $\sin \gamma=\operatorname{sech} \beta=R e^{-i \theta}$, then $0<R e(\sin \gamma)<1$. Therefore, the Theorem will hold for complex values of $\gamma$ such that $0<\operatorname{Re}(\cos \gamma)<1$ and $0<\operatorname{Re}(\sin \gamma)<1$.

\section{Appendix}

The formula (2.1) resembles Sonine's integral discussed in [4, p. 416], but it is necessary to establish it independently. It is convenient to consider first the integral

$$
J=\int_{0}^{\infty} K_{i s}(x) K_{0}\left[\left(x^{2}+t^{2}\right)^{\frac{1}{2}}\right] d x .
$$

On inserting the expression $[1$, p. 86$]$,

$$
K_{0}\left[\left(x^{2}+t^{2}\right)^{\frac{1}{2}}\right]=\int_{0}^{\infty} e^{-t \cosh v} \cos (x \sinh v) d v,
$$

it follows that

$$
J=\int_{0}^{\infty} K_{i s}(x) \int_{0}^{\infty} e^{-t \cosh v} \cos (x \sinh v) d v d x .
$$

Since $\left|K_{i s}(x)\right| \leq K_{0}(x)$, it is seen that the repeated integral (A.2) is absolutely convergent since its magnitude cannot exceed the quantity

$$
\int_{0}^{\infty} K_{0}(x) d x \int_{0}^{\infty} e^{-t \cosh v} d v
$$

On reversing the order of integration in (A.2) and appealing to the formula [1, p. 412]

$$
\int_{0}^{\infty} K_{i s}(x) \cos (x \sinh v) d x=\frac{1}{2} \pi \operatorname{sech} v \operatorname{sech}\left(\frac{1}{2} s \pi\right) \cos (s v),
$$

it is found that

$$
\begin{aligned}
J & =\frac{1}{2} \pi \operatorname{sech}\left(\frac{1}{2} s \pi\right) \int_{0}^{\infty} e^{-t \cosh v} \operatorname{sech} v \cos (s v) d v \\
& =\frac{1}{2} \pi \operatorname{sech}\left(\frac{1}{2} s \pi\right) \int_{0}^{\infty} \cos (s v) \int_{t}^{\infty} e^{x \cosh v} d x d v .
\end{aligned}
$$

This repeated integral also is absolutely convergent, and on reversing the order of integration and recalling the definition (1.2) of $K_{i s}(x)$, it is found that

$$
J=\frac{1}{2} \pi \operatorname{sech}\left(\frac{1}{2} s \pi\right) \int_{t}^{\infty} K_{i s}(x) d x .
$$

On calculating $d J / d t$ from (A.1) and (A.3) and equating the resulting expressions, we obtain, since $K_{0}^{\prime}(x)=-K_{1}(x)$, the equation

$$
\int_{0}^{\infty} t\left(x^{2}+t^{2}\right)^{-\frac{1}{2}} K_{i s}(x) K_{1}\left[\left(x^{2}+t^{2}\right)^{\frac{1}{2}}\right] d x=\frac{1}{2} \pi \operatorname{sech}\left(\frac{1}{2} s \pi\right) K_{i s}(t) .
$$


The differentiation of (A.1) is in order since the application of a Dirichlet-type criterion shows that the integral on the left-hand side of (A.4) is uniformly convergent for all $t \geq 0$. Since the factor $t\left(x^{2}+t^{2}\right)^{-\frac{1}{2}} K_{1}\left[\left(x^{2}+t^{2}\right)^{\frac{1}{2}}\right]$ is a non-increasing function of $x$ that tends to zero as $x \rightarrow \infty$ uniformly for all $t \geq 0$, (A.4) is established.

Expressions for the coefficients $h_{0}, h_{2}, h_{4}$ appearing in (3.12) are given by the following formulas. The formula for $h_{4}(m, n)$ is given only for $m=n=0$ since the expression for general values of $m, n$ is somewhat cumbersome:

$$
\begin{aligned}
& h_{0}(m, n)= 2^{-\frac{1}{2}}, \\
& h_{2}(m, n)=2^{-\frac{1}{2}}[(n+c)(n+c-1) \operatorname{coth}^{2} \beta+\left(m+\frac{1}{2}\right)\left(m+\frac{3}{2}\right) \tanh ^{2} \beta \\
&\left.\quad+2 m(n+c)-3 m-\frac{3}{4}\right], \\
& h_{4}(0,0)=2^{-\frac{1}{2}}\left[\frac{1}{2}\left(c-\frac{35}{8}\right) \tanh ^{2} \beta+\frac{35}{32} \tanh ^{4} \beta-\frac{1}{3} c(c-1)\left(2 c+\frac{11}{4}\right) \operatorname{coth}^{2} \beta\right. \\
&\left.\quad+\frac{1}{6} c\left(c^{2}-1\right)(c+2) \operatorname{coth}^{4} \beta+\frac{1}{4}(c-1)(c-2)+\frac{19}{32}\right] .
\end{aligned}
$$

Finally, the connection between $f$ and $g$ is examined. To do this, we introduce the function

$$
g_{1}(x)=\int_{0}^{\infty} f(t) K_{0}\left[\left(x^{2}+t^{2}\right)^{\frac{1}{2}}\right] d t
$$

so that $g(x)=-d g_{1} / d x$, and appeal to the equation [1, pp. 402 and 447$]$

$$
\int_{0}^{\infty} K_{0}\left[\left(x^{2}+t^{2}\right)^{\frac{1}{2}}\right] \cos (s x) d x=\frac{\pi}{2} \int_{t}^{\infty} e^{-s x} J_{0}\left[\left(x^{2}-t^{2}\right)^{\frac{1}{2}}\right] d x .
$$

It follows from this result and (A.5) that

$$
\int_{0}^{\infty} g_{1}(x) \cos (s x) d x=\frac{\pi}{2} \int_{0}^{\infty} e^{-s x} f_{1}(x) d x
$$

where

$$
f_{1}(x)=\int_{0}^{x} f(t) J_{0}\left[\left(x^{2}-t^{2}\right)^{\frac{1}{2}}\right] d t .
$$

If $g_{1}(x)$ is supposed given, the equation (A.6) yields the Laplace transform of $f_{1}(x)$, from which $f_{1}(x)$ can be found. Let $I(s)$ denote the function on the left-hand side of (A.6). Then according to Widder [5, p. 315], the representation of this function as a Laplace transform exists if $\left|I^{(k)}(s)\right|<M(k !) s^{-k-1}$ for $k=0,1,2 \ldots$, where $M$ is some constant. In the present case, it is readily verified by calculating $I^{(k)}(s)$, integrating by parts $k$ times, and noting that $x^{m+1} g_{1}^{(m)}(x) \rightarrow 0$ as $x \rightarrow 0$ for $m=0,1,2, \ldots$, that Widder's conditions are satisfied so that $f_{1}(x)$ exists. Finally, $f(t)$ can be found from the equation (A.7) since the latter is a simple type of convolution integral whose solution is given by the equation

$$
f(x)=x^{-2}\left(x \frac{d^{2}}{d x^{2}}-\frac{d}{d x}\right) \int_{0}^{x} f_{1}(t) I_{0}\left[\left(x^{2}-t^{2}\right)^{\frac{1}{2}}\right] t d t .
$$

Acknowledgment. The author wishes to thank the authorities at University College, London, for permission to use the facilities of the college during the writing of this paper. 


\section{References}

1. W. Magnus, F. Oberhettinger and R. P. Soni, Formulas and Theorems for the Special Functions of Mathematical Physics, Springer-Verlag, New York, 1965.

2. D. Naylor, On an Asymptotic Expansion of the Kontorovich-Lebedev Transform, Applicable Analysis 39 (1990), 249-264.

3. F. W. J. Olver, Asymptotics and Special Functions, Academic Press, New York, 1974.

4. G. N. Watson, Theory of Bessel Functions, 2nd ed., Cambridge University Press, Cambridge, 1958.

5. D. V. Widder, The Laplace Transform, Princeton University Press, Princeton, 1946.

6. R. Wong, Asymptotic Expansions of the Kontorovich-Lebedev Transform, Applicable Analysis 12 (1980), 161-172.

Department of Applied Mathematics, University of Western Ontario, London, Ontario, Canada N6A 5B7 